\title{
The Lower Bound of Density Estimation for Biased Data in Sobolev Spaces
}

\author{
Jinru Wang ${ }^{1, a} \quad$ Yuan Zhou ${ }^{2, b}$ \\ ${ }^{12}$ College of Applied Science, Beijing University of Technology, Beijing, China \\ awangjinru@bjut.edu.cn byuanzhou@emails.bjut.edu.cn
}

Keywords: Lower Bound, Density Estimation, Biased Data, Sobolev Spaces.

\begin{abstract}
In this paper, we consider the density estimation problem from independent and identically distributed (i.i.d.) biased observations. We study the lower bound of convergence rates of density estimation over Sobolev spaces $W_{\mathrm{r}}^{\mathrm{N}}\left(N \in N^{+}\right)$under the $L_{p}$ risk by using Fano's lemma.
\end{abstract}

\section{Introduction}

In this paper, we consider the problem of estimating the density functions without observing directly the sample $X_{1}, \ldots X_{\mathrm{n}}$. We observe an i.i.d. sample $Y_{1}, \ldots Y_{\mathrm{n}}$ from a biased distribution with the following density function $f^{\mathrm{Y}}(y)=g(y) f^{\mathrm{X}}(y) / \mu$, where $\mathrm{g}(\mathrm{y})$ is the so-called weighting or biasing function, $\mu=E \mathrm{~g}(X)$. Our purpose is to estimate the density function $f^{\mathrm{X}}$ from $Y_{1}, Y_{2}, \ldots Y_{\mathrm{n}}$. Several examples about this biased data can be found in the literature. For instance, in paper [1], it is shown that the distribution of the concentration of alcohol in the blood of intoxicated drivers is of interest, since the drunken driver has a larger chance of being arrested, the collected data are size-biased.

For unbiased data, Kerkyacharian and Picard [2] study a Besov space with matched case. Donoho, Johnstone, Kerkyacharian and Picard [3] consider a Besov space with unmatched case. They show the lower bound by using Korostelev and Assouad lemmas. However, the conditions of those two lemmas are difficult to be verified. In 2011, Huiying Wang [4] give a proof by using Fano's lemma. In reference [3, 4], they show the lower bound of convergence rates over Besov for $L_{p}$ risk.

So far, wavelet density estimations about the lower bound of convergence rates over Besov spaces have made some achievements. However, there is a few people to study density estimation in Sobolev spaces $W_{\mathrm{r}}^{\mathrm{N}}\left(N \in N^{+}\right)$. In this paper, we study the lower bound of density estimation convergence over Sobolev spaces.

\section{Preliminaries}

In this paper, we always assume that scaling wavelet $\varphi(x)$ is orthonormal, compactly supported and $N+1$ regular. We consider the Sobolev balls $\tilde{W}_{r}^{N}(A, L)$ which is defined by:

$$
\tilde{W}_{r}^{N}(A, L):=\left\{f: f \in W_{r}^{N}(R), f \text { is a probability density on } R\right. \text { with a compact support of }
$$
length $\leq A$, and $\left.\left\|f^{(N)}\right\|_{r} \leq L\right\}$.

Lemma 1 (Fano's lemma, [5]) Let $\left(\Omega, X, P_{k}\right)$ be probability measurable spaces and $A_{k} \in X, k=0,1, \ldots, m$. If $A_{k} \cap A_{v}=\Phi$ for any $k \neq v, A_{k}^{c}$ standing for the complement of $A_{k}$ and $k_{m}:=\inf _{0 \leq v \leq m} \frac{1}{m} \sum_{k \neq v} K\left(P_{k}, P_{v}\right)$, we have

$$
\sup _{0 \leq k \leq m} P_{k}\left(A_{k}^{c}\right) \geq \min \left\{\frac{1}{2}, \sqrt{m} \exp \left(-3 e^{-1},-k_{m}\right)\right\} .
$$

Lemma 2 (Varshamov-Gilbert lemma, [5]) Let $\Theta:=\left\{\varepsilon=\left(\varepsilon_{1}, \cdots, \mathcal{E}_{m}\right)\right\}, \mathcal{E}_{i} \in 0,1$, then there exists a 
subset $\left\{\varepsilon^{0}, \cdots, \varepsilon^{M}\right\}$ of $\Theta$ with $\varepsilon^{0}=(0, \cdots, 0)$ such that $M \geq 2^{m / 8}$, and $\sum_{k=1}^{m}\left|\varepsilon_{k}^{i}-\varepsilon_{k}^{j}\right| \geq \frac{m}{8}(0 \leq i \neq j \leq M)$.

\section{Main result}

Theorem 3 Let $\varphi$ be a compactly supported, $N+1$ regular and orthonormal scaling function, $f^{X} \in \tilde{W}_{r}^{N}(A, L)$. We assume that there exist two constants $g_{1}$ and $g_{2}$ such that, for any $x \in R, 0<g_{1} \leq g(x) \leq g_{2}<\infty$. If $\hat{f}_{n}^{X}(x)$ is any estimator of $f^{X}$, then for $\forall 1 \leq r, p<\infty, N>1 / r$, we have

$$
\sup _{f^{X} \in \tilde{W}_{r}^{N}(A, L)} E\left\|\hat{f}_{n}^{X}(x)-f^{X}(x)\right\|_{p} \succ \max \left\{\left(\frac{\ln n}{n}\right)^{\frac{N-1 / r+1 / p}{2(N-1 / r)+1}}, n^{-\frac{N}{2 N+1}}\right\} .
$$

where $x \succ y$ means $x \geq c y$ with a positive constant $c$.

Proof of Theorem 3: Using the idea of reference[4], firstly, we prove

$$
\sup _{f^{X} \in \widetilde{W}_{r}^{N}(A, L)} E\left\|\hat{f}_{n}^{X}(x)-f^{X}(x)\right\|_{p} \succ\left(\frac{\ln n}{n}\right)^{\frac{N-1 / r+1 / p}{2(N-1 / r)+1}} .
$$

Now we construct $h_{k}(x)$, such that $h_{k}(x) \in \tilde{W}_{r}^{N}(A, L)$ and

$$
\sup _{k} E\left\|\hat{f}_{n}^{X}(x)-h_{k}(x)\right\|_{p} \succ\left(\frac{\ln n}{n}\right)^{\frac{N-1 / r+1 / p}{2(N-1 / r)+1}} .
$$

Let $\varphi$ be a compactly supported, $N+1$ regular and orthonormal scaling function, $\psi$ be the corresponding wavelet, and $\operatorname{supp} \psi \in[0, l), l<A$. Then there exists a compactly supported density function $h(x)$ satisfying $h(x) \in W_{r}^{N}(R)$, and $\left.h(x)\right|_{[0, l)}=C_{0}>0$.

Denote $\Delta_{j}=\left\{0, l, 2 l, \ldots,\left(2^{j}-1\right) l, 2^{j} l\right\}$, then the number of elements in $\Delta_{j}$ is $2^{j}+1$. We define $a_{j}=2^{-j(N+1 / 2-1 / r)}, h_{k}(x)=h(x)+a_{j} \psi_{j, k}(x) I\left\{k \neq 2^{j} l\right\}, k \in \Delta_{j}$. Obviously we have $h_{2^{j} l}(x)=h(x)$, $h_{k}(x) \in \tilde{W}_{r}^{N}(A, L)$. Let $h_{k}^{Y}(x)$ be the density function of $Y_{1}, Y_{2}, \cdots, Y_{n}$, then $h_{k}^{Y}(x)=\frac{g(x) h_{k}(x)}{\mu}$. For any $k \neq k^{\prime}$, we get

$$
\left\|h_{k}-h_{k^{\prime}}\right\|_{p}=a_{j}\left\|\psi_{j, k}(x)-\psi_{j, k^{\prime}}(x)\right\|_{p} \geq a_{j}\left\|\psi_{j, k}\right\|_{p}=2^{-j(N+1 / p-1 / r)}\|\psi\|_{p}:=\eta_{j} .
$$

If denote $A_{k}=\left\{\left\|\hat{f}_{n}^{X}-h_{k}\right\|_{p}<\frac{\eta_{j}}{2}\right\}$, then using Fano's Lemma, we have

$$
\sup _{k \in \Delta_{j}} P_{h_{k}^{Y}}^{n}\left(A_{k}^{c}\right) \geq \min \left\{\frac{1}{2}, \sqrt{2^{j}} \exp \left(-3 e^{-1}-k_{2^{j}}\right)\right\},
$$

where $P_{f}^{n} \quad$ stands for the probability measure corresponding to the density function $f^{n}(x)=f\left(x_{1}\right) f\left(x_{2}\right) \cdots f\left(x_{n}\right)$. Since

$$
E\left\|\hat{f}_{n}^{X}(x)-h_{k}(x)\right\|_{p} \geq \frac{\eta_{j}}{2} P_{h_{k}^{x}}^{n}\left(\left\|\hat{f}_{n}^{X}-h_{k}\right\|_{p} \geq \frac{\eta_{j}}{2}\right)=\frac{\eta_{j}}{2} P_{h_{k}^{Y}}^{n}\left(A_{k}^{c}\right),
$$

then $\sup _{k \in \Delta_{j}} E\left\|\hat{f}_{n}^{X}(x)-h_{k}(x)\right\|_{p} \geq \sup _{k \in \Delta_{j}} \frac{\eta_{j}}{2} P_{h_{k}^{x}}^{n}\left(A_{k}^{c}\right) \geq \frac{\eta_{j}}{2} \min \left\{\frac{1}{2}, \sqrt{2^{j}} \exp \left(-3 e^{-1}-k_{2^{j}}\right)\right\}$.

where $k_{2^{j}}:=\inf _{v \in \Delta_{j}} \frac{1}{2^{j}} \sum_{k \neq v} K\left(P_{h_{k}^{Y}}^{n}, P_{h_{v}^{Y}}^{n}\right)$. Next, we shows $k_{2^{j}} \leq g_{2} C_{0}^{-1} n a_{j}^{2} / g_{1}$. From 


$$
K\left(P_{f_{1}}^{n}, P_{f_{2}}^{n}\right)=\int_{f_{1}^{n} \cdot f_{2}^{n}>0} f_{1}^{n}(x) \ln \frac{f_{1}^{n}(x)}{f_{2}^{n}(x)} d x=\sum_{j=1}^{n} \int f_{1}\left(x_{j}\right) \ln \frac{f_{1}\left(x_{j}\right)}{f_{2}\left(x_{j}\right)} d x_{j}=n K\left(P_{f_{1}}^{1}, P_{f_{2}}^{1}\right),
$$

and for any $u>0, \ln u \leq u-1$, we have

and

$$
\begin{aligned}
& K\left(P_{f_{1}}^{n}, P_{f_{2}}^{n}\right)=n K\left(P_{f_{1}}^{1}, P_{f_{2}}^{1}\right) \leq n \int f_{1}(x)\left(\frac{f_{1}(x)}{f_{2}(x)}-1\right) d x=n \int f_{2}^{-1}(x)\left(f_{1}(x)-f_{2}(x)\right)^{2} d x, \\
& k_{2^{j}} \leq 2^{-j} \sum_{k \in \Delta_{j}, k \neq 2^{j} l} \int\left(\frac{g(x) h_{2^{j} l}(x)}{\mu}\right)^{-1}\left(\frac{g(x) h_{k}(x)}{\mu}-\frac{g(x) h_{2^{j} l}(x)}{\mu}\right)^{2} d x
\end{aligned}
$$

$$
\leq \frac{g_{2}}{g_{1}} 2^{-j} n \sum_{k \in \Delta_{j}, k \neq 2^{j} l} C_{0}^{-1} \int a_{j}^{2}\left|\psi_{j, k}\right|^{2} d x=g_{2} n C_{0}^{-1} a_{j}^{2} / g_{1}
$$

Taking $2^{j} \sim\left(\frac{n}{\ln n}\right)^{\frac{1}{2(N-1 / r)+1}}$, then $n a_{j}^{2}=n 2^{-2 j(N+1 / 2-1 / r)} \sim n\left(\frac{n}{\ln n}\right)^{-1}=\ln n$.

We choose $C$ such that $n a_{j}^{2} \leq C \ln n, C g_{2}[4(N-1 / r)+2]<C_{0} g_{1}$, then

$$
\sqrt{2^{j}} e^{-k_{2 j}} \geq \sqrt{2^{j}} e^{-g_{2} C_{0}^{-1} n a_{j}^{2} / g_{1}} \geq \sqrt{2^{j}} n^{-g_{2} C_{0}^{-1} C / g_{1}} \geq 1 \text {. }
$$

Hence, $\sup _{k \in \Delta_{j}} E\left\|\hat{f}_{n}^{X}(x)-h_{k}(x)\right\|_{p} \geq \frac{\eta_{j}}{2} \min \left\{\frac{1}{2}, \sqrt{2^{j}} \exp \left(-3 e^{-1}-k_{2^{j}}\right)\right\} \geq C \eta_{j}$. Since

$\eta_{j}=2^{-j(N+1 / p-1 / r)}\|\psi\|_{p} \sim C\left(\frac{\ln n}{n}\right)^{\frac{N+1 / p-1 / r}{2(N-1 / r)+1}}$, then

$$
\sup _{k \in \Delta_{j}} E\left\|\hat{f}_{n}^{X}(x)-h_{k}(x)\right\|_{p} \succ\left(\frac{\ln n}{n}\right)^{\frac{N+1 / p-1 / r}{2(N-1 / r)+1}} .
$$

Therefore, $\sup _{f^{X} \in \tilde{W}_{r}^{N}(A, L)} E\left\|\hat{f}_{n}^{X}(x)-f^{X}(x)\right\|_{p} \succ\left(\frac{\ln n}{n}\right)^{\frac{N-1 / r+1 / p}{2(N-1 / r)+1}}$.

Next, we prove $\sup _{f^{X} \in \tilde{W}_{r}^{N}(A, L)} E\left\|\hat{f}_{n}^{X}(x)-f^{X}(x)\right\|_{p} \succ n^{-\frac{N}{2 N+1}}$. Defining

$$
a_{j}=2^{-j(N+1 / 2)}, \quad h_{\varepsilon^{i}}(x)=h(x)+a_{j} \sum_{k \in \Delta_{j}} \varepsilon_{k}^{i} \psi_{j, k}(x), i=0,1, \cdots, M .
$$

with $\varepsilon^{i}=\left(\varepsilon_{k}^{i}\right)_{k \in \Delta_{j}}, \varepsilon_{k}^{i} \in\{0,1\}$. Obviously $h_{\varepsilon^{0}}(x)=h(x)$. Since

$h_{\varepsilon^{i}}(x)=h(x)+a_{j} \sum_{k \in \Delta_{j}} \varepsilon_{k}^{i} \psi_{j, k}(x) \geq C_{0}-a_{j} \sum_{k \in \Delta_{j}}\left\|\psi_{j, k}\right\|_{\infty} \geq C_{0}-2^{-j(N-1)}\|\psi\|_{\infty} \geq 0$ (for large $j$ ), so $h_{\varepsilon^{i}}(x)$ is a probability density function. By the assumptions of $\varphi$, the wavelet $\psi$ is compactly supported and $N+1$ times differentiable. Therefore $\psi \in \tilde{W}_{r}^{N}$, and we get

$$
\left\|h_{\varepsilon^{i}}^{(N)}(x)\right\|_{r} \leq\left\|h^{(N)}(x)\right\|_{r}+\left\|a_{j} \sum_{k \in \Delta_{j}} \varepsilon_{k}^{i} \psi_{j, k}^{(N)}(x)\right\|_{r} .
$$

Since $\forall k \neq k^{\prime}$, supp $\psi_{j k} \cap \operatorname{supp} \psi_{j k^{\prime}}=\Phi$, then we get

$$
\left\|a_{j} \sum_{k \in \Delta_{j}} \varepsilon_{k}^{i} \psi_{j, k}^{(N)}(x)\right\|_{r}=a_{j}\left(\int\left|\sum_{k \in \Delta_{j}} \varepsilon_{k}^{i} \psi_{j, k}^{(N)}(x)\right|^{r} d x\right)^{1 / r}=a_{j} 2^{j(1 / 2-1 / r)} 2^{j N}\left(\sum_{k \in \Delta_{j}}\left|\varepsilon_{k}^{i}\right|^{r}\right)^{1 / r}\left\|\psi^{(N)}\right\|_{r} .
$$

From $a_{j}=2^{-j(N+1 / 2)}$, and $\sum_{k \in \Delta_{j}}\left|\varepsilon_{k}^{i}\right|^{r} \leq 2^{j}$, we have 


$$
\left\|a_{j} \sum_{k \in \Delta_{j}} \varepsilon_{k}^{i} \psi_{j, k}^{(N)}(x)\right\|_{r} \leq\left\|\psi^{(N)}\right\|_{r}
$$

hence, $\left\|h_{\varepsilon^{i}}^{(N)}(x)\right\|_{r} \leq L$. Therefore $h_{\varepsilon^{i}}(x) \in \tilde{W}_{r}^{N}(A, L)$.

Let $h_{\varepsilon^{i}}^{Y}(x)$ be the density function of $Y_{1}, Y_{2}, \cdots, Y_{n}$, then $h_{\varepsilon^{i}}^{Y}(x)=\frac{g(x) h_{\varepsilon^{i}}(x)}{\mu}$. By Varshamov-Gilbert lemma, there exists $\left\{\varepsilon^{0}, \cdots, \varepsilon^{M}\right\}$ with $\varepsilon^{0}=(0, \cdots, 0)$, such that $M \geq 2^{2^{j / 8}}$ and $\sum_{k=1}^{m}\left|\varepsilon_{k}^{i}-\varepsilon_{k}^{l}\right| \geq \frac{2^{j}}{8}(0 \leq i \neq l \leq M)$. For any $i \neq l$,

$$
\left\|h_{\varepsilon^{i}}-h_{\varepsilon^{l}}\right\|_{p}^{p}=\left\|a_{j} \sum_{k \in \Delta_{j}}\left(\varepsilon_{k}^{l}-\varepsilon_{k}^{i}\right) \psi_{j, k}\right\|_{p}^{p} \geq\|\psi\|_{p}^{p} 2^{-(p N+1) j} 2^{j-3}=\|\psi\|_{p}^{p} 2^{-p j N} 2^{-3},
$$

we have $\left\|h_{\varepsilon^{i}}-h_{\varepsilon^{\prime}}\right\|_{p} \geq\|\psi\|_{p} 2^{-N j} 8^{-1 / p}:=\eta_{j}$. If we let

$$
A_{\varepsilon^{i}}=\left\{\left\|\hat{f}_{n}^{X}-h_{\varepsilon^{i}}\right\|_{p}<\frac{\eta_{j}}{2}\right\}, \quad i=0,1, \ldots, M,
$$

then $\forall i \neq l$, we get $A_{\varepsilon^{i}} \cap A_{\varepsilon^{\prime}}=\Phi$. Using Fano's Lemma, we have

$$
\sup _{i} P_{h_{\varepsilon^{\prime}}^{r}}^{n}\left(A_{\varepsilon^{i}}^{c}\right) \geq \min \left\{\frac{1}{2}, \sqrt{M} \exp \left(-3 e^{-1}-k_{M}\right)\right\} \text {. }
$$

and $E\left\|\hat{f}_{n}^{X}(x)-h_{\varepsilon^{i}}(x)\right\|_{p}=\frac{\eta_{j}}{2} P_{h_{\varepsilon^{i}}^{x}}^{n}\left(A_{k}^{c}\right)$. Moreover,

$$
\sup _{k \in \Delta_{j}} E\left\|\hat{f}_{n}^{X}(x)-h_{\varepsilon^{i}}(x)\right\|_{p} \geq \sup _{k \in \Delta_{j}} \frac{\eta_{j}}{2} P_{h_{\varepsilon^{i}}^{Y}}^{n}\left(A_{k}^{c}\right) \geq \frac{\eta_{j}}{2} \min \left\{\frac{1}{2}, \sqrt{M} \exp \left(-3 e^{-1}-k_{M}\right)\right\} .
$$

where $k_{M}:=\inf _{0 \leq v \leq M} \frac{1}{M} \sum_{i \neq v} K\left(P_{h_{e}^{\gamma}}^{n}, P_{h_{e^{\nu}}^{v}}^{n}\right)$. Next, we shows $k_{M} \leq g_{2} C_{0}^{-1} n a_{j}^{2} 2^{j} / g_{1}$. We compute that

$$
\begin{aligned}
k_{M} & =\inf _{0 \leq v \leq M} \frac{1}{M} \sum_{i \neq v} K\left(P_{h_{\varepsilon^{Y}}^{Y}}^{n}, P_{h_{\varepsilon^{V}}^{Y}}^{n}\right) \leq \frac{1}{M} \sum_{0<i \leq M} K\left(P_{h_{\varepsilon^{\prime}}^{Y}}^{n}, P_{\xi_{\varepsilon^{Y}}^{Y}}^{n}\right) \\
& \leq \frac{n}{M} \sum_{0<i \leq M} \int\left(\frac{g(x) h(x)}{\mu}\right)^{-1}\left(\frac{g(x) h_{\varepsilon^{i}}(x)}{\mu}-\frac{g(x) h(x)}{\mu}\right)^{2} d x \\
& \leq \frac{g_{2}}{g_{1}} \frac{n}{M} \sum_{0<i \leq M} C_{0}^{-1} a_{j}^{2} \sum_{k \in \Delta_{j}} \int\left|\psi_{j, k}(x)\right|^{2} d x=g_{2} n C_{0}^{-1} a_{j}^{2} 2^{j} / g_{1}
\end{aligned}
$$

Taking $2^{j} \sim n^{\frac{1}{2 N+1}}$, then $n a_{j}^{2}=n 2^{-j(2 N+1)} \sim 1$. We choose $C$ such that $n a_{j}^{2} \leq C$, and $C<C_{0} g_{1} / 32 g_{2}$. From $\sqrt{M} \geq \sqrt{2^{2^{j-3}}}$, we get

$$
\sqrt{M} e^{-k_{M}} \geq 2^{2^{j-4}} e^{-g_{2} C_{0}^{-1} n a_{j}^{2} 2^{j} / g_{1}} \geq 2^{2^{j-4}\left(1-32 C g_{2} C_{0}^{-1} g_{1}^{-1}\right)} \geq 1 .
$$

So $\quad \sup _{k \in \Delta_{j}} E\left\|\hat{f}_{n}^{X}(x)-h_{\varepsilon^{i}}(x)\right\|_{p} \geq \sup _{k \in \Delta_{j}} \frac{\eta_{j}}{2} P_{h_{\varepsilon^{i}}}^{n}\left(A_{k}^{c}\right) \geq c \eta_{j}, \quad$ and $\quad \eta_{j}=\|\psi\|_{p} 2^{-N j} 8^{-1 / p} \sim n^{-\frac{N}{2 N+1}}$, therefore $\sup _{f^{X} \in \widetilde{W}_{r}^{N}(A, L)} E\left\|\hat{f}_{n}^{X}(x)-f^{X}(x)\right\|_{p} \succ n^{-\frac{N}{2 N+1}}$.

\section{Acknowledgements}

This paper is supported by Beijing Educational Committee Foundation (No.PHR201008022) and 
Fundamental Research Foundation of Beijing University of Technology.

\section{References}

[1] S. Efromovich, Nonparametric curve estimation. Methods, Theory, and Applications, Springer Series in Statistics, 1999.

[2] G. Kerkyacharian, D. Picard. Density estimation in Besov spaces. Statist. Proab. Lett., 13 (1992 ) $15-24$.

[3] D. L. Donoho, I. M.Johnstone, G. Kerkyacharian, D. Picard. Density estimation by wavelet thresholding. Ann. Statist., 24 (1996) 508-539.

[4] H. Y. Wang. Convergence rates of density estimation in Besov spaces. Applied Mathematics, 2 (2011) 1258-1262.

[5] A. B. Tsybakov. Introduction to Nonparametric Estimation. Springer-Verlag, Berlin, 2009.

[6] S. Efromovich, Density estimation for biased data. The Annals of Statistics, 32 (2004) 1137-1161.

[7] C. Chesnean. Wavelet block thresholding for density estimation in the presence of bias. Journal of the Korean Statistical Society, 39 (2010) 43-53. 